\title{
COMPORTAMENTO TÉRMICO DE LODOS DE ESGOTO SANITÁRIO EM ATMOSFERA INERTE E OXIDANTE
}

\author{
L. BATISTELLA ${ }^{1}$, V.F. DA SILVA ${ }^{1}$, T.R. PACIONI ${ }^{1}$, M. MENEZES ${ }^{1}$, C.A. ALTHOFF ${ }^{2}$, H.J. \\ JOSÉ $^{1}$
}

${ }^{1}$ Universidade Federal de Santa Catarina, Departamento de Engenharia Química e Engenharia de Alimentos.

${ }^{2}$ Albrecht Indústria de Equipamentos Ltd., BR-101, km 29, Pirabeiraba, Joinville, SC, Brazil

E-mail para contato: batistella.luciane@gmail.com

RESUMO - O lodo de esgoto é o principal subproduto gerado no tratamento de águas residuais urbanas e representa um desafio quanto a sua disposição final. O objetivo deste trabalho foi caracterizar e avaliar o comportamento térmico de quatro amostras de lodos (anaeróbios e aeróbios) em atmosfera inerte e oxidante. O poder calorífico inferior dos lodos variou entre 7,58 MJ.Kg ${ }^{-1}$ e $14,55 \mathrm{MJ}^{-} \mathrm{Kg}^{-1}$ e a matéria volátil entre $44,68 \%$ e $63,50 \%$, indicando o potencial energético deste combustível e viabilizando seu uso como fonte alternativa de energia. Foram observados dois comportamentos térmicos diferentes de acordo com o tratamento dos lodos. Para as amostras de lodos anaeróbios os perfis termogravimétricos de pirólise são idênticos aos de combustão. Para o lodo aeróbio, os perfis termogravimétricos de pirólise e combustão coincidem, exceto na última parte do perfil de combustão, onde uma decomposição final aparece como consequência da combustão do char formado anteriormente. O comportamento diferente está associado a estabilização anaeróbia e aeróbia que os lodos sofre durante o tratamento nas estações de tratamento de esgotos.

\section{INTRODUÇÃO}

O lodo é o principal resíduo gerado durante o tratamento mecânico, biológico e químico dos esgotos. Este produto é constituído por matéria orgânica (hidrocarbonetos, aminoácidos, proteínas, gorduras, celulose, microrganismos), e uma grande variedade de substâncias inorgânicas. Devido, ao crescimento da população das cidades e a melhoria nos sistemas de tratamento de efluentes a quantidade de lodo de esgoto gerado tem aumentado, representando um desafio quanto a sua disposição (Rulkens, 2008).

Como lodo tem relativamente alto "valor calórico", as tecnologias de valorização energética, como a combustão, pirólise, gaseificação e co-combustão tem ganhado atenção Na combustão ocorre à completa oxidação dos compostos orgânicos do lodo a altas temperaturas liberando $\mathrm{CO}_{2}$ e água. $\mathrm{O}$ volume dos lodos é reduzido e é gerada energia. No processo de pirólise os lodos são aquecidos a uma temperatura de $350-500{ }^{\circ} \mathrm{C}$ na ausência de oxigênio, gerando char, óleos de pirólise, vapor de água, cinzas e gases combustíveis. O combustível que é produzido pode ser usado em motores de 


\section{9 a 22 de outubro de 2014 \\ Florianópolis/SC}

combustão (Gómez-Rico, et al., 2005; Rulkens, 2008).

As características da pirólise e combustão dos lodos de esgoto têm sido extensivamente estudadas por técnicas térmicas como TG, DTG e DTA. As vantagens da análise termogravimétrica incluem a rápida avaliação do valor do combustível, a determinação das temperaturas de inicio e termino dos processos, a temperatura de máxima reatividade, entre outras. (Magdziarz e Wilk, 2013) Portanto, este estudo foi realizado com o objetivo de prever o comportamento dos lodos durante o processo de combustão e pirólise por meio de ensaios termogravimétricos. Estes dados serão úteis para a definição e ajuste das condições do processo que será realizado em escala piloto, especialmente a temperatura a combustão que, por sua vez, regula as propriedades das cinzas.

\section{MATERIAIS E MÉTODOS}

\subsection{Lodos selecionados}

Foram selecionadas cinco amostras de lodos de esgoto secos provenientes de diferentes tipos de tratamento de esgotos sanitários, que estão mostrados na Tabela 1.

Tabela 1. Tipos de lodos e tratamentos.

\begin{tabular}{ll}
\hline Lodo & \multicolumn{1}{c}{ Tratamento } \\
\hline LSC & Processo anaeróbio: reatores UASB (sigla em ingles: Up flow anaerobic sludge blanket) \\
LSAE & Processo aeróbio: lodos ativados \\
LSAN & Processo anaeróbio: reatores UASB \\
LSANF & Lodo dos reatores UASB misturado com lodo do sistema de flotação (adensamento) \\
LSANS & Lodo dos reatores UASB misturado com escuma gerada nos reatores UASB \\
\hline
\end{tabular}

\subsection{Caracterização dos Lodos}

Análise Elementar, Imediata e Poder Calorífico: Para a análise elementar de $\mathrm{C}, \mathrm{H}$ e $\mathrm{N}$, aproximadamente 1,0 mg da amostra foi introduzida no equipamento (Elemental Analyzer $2400 \mathrm{CHN}$ Perkin-Elmer Series II - USA) sendo submetida à combustão a $925^{\circ} \mathrm{C}$ na presença de oxigênio puro. Durante a análise, o carbono passa para a forma de $\mathrm{CO}_{2}$, o hidrogênio para a forma de $\mathrm{H}_{2} \mathrm{O}$ e o nitrogênio formará vários óxidos que, ao passar por uma coluna contendo cobre metálico $\left(640{ }^{\circ} \mathrm{C}\right)$, é reduzido para $\mathrm{N}_{2}$. Os gases resultantes, $\mathrm{CO}_{2}, \mathrm{H}_{2} \mathrm{O}$ e $\mathrm{N}_{2}$ são arrastados pelo gás hélio, homogeneizados e posteriormente separados através de colunas específicas (empacotadas com sílica) e detectados por um detector de condutividade térmica (TCD).

Para determinar a concentração de enxofre e cloro foi utilizada a técnica de combustão em frasco de Shoniger (erlenmeyer hermeticamente fechado). Neste procedimento, a amostra solida é envolvida por um material, geralmente papel filtro, posicionado em uma alça de platina. $O$ frasco é preenchido com oxigênio à pressão atmosférica e o papel filtro contendo a amostra é inserido no interior do frasco. A ignição é feita de maneiras manual e depois o frasco é inclinado para que a solução absorvedora vede completamente o frasco, evitando perdas do analito. Na determinação do enxofre, 15,0 mg de 


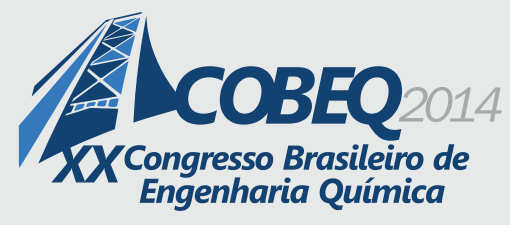

19 a 22 de outubro de 2014
Florianópolis/SC

lodo foram queimadas no frasco de Schoniger em temperaturas superiores a $600{ }^{\circ} \mathrm{C}$. O líquido absorvente utilizando foi uma solução de $\mathrm{H}_{2} \mathrm{O}_{2}$. O frasco foi mantido fechado durante um período de 3 horas, para que todo $\mathrm{SO}_{4}$ formado seja absorvido pelo líquido absorvente. Posteriormente a concentração de $\mathrm{S}$ foi determinada por meio da análise da solução por ICP-AES (Arcos SOP USA 2010). As mesmas condições de queima foram utilizadas para determinar a concentração de cloro nos lodos, diferenciando apenas na solução absorvedora $(\mathrm{NaOH})$. A solução foi titulada com de nitrato de mercúrio $\left(\left(\mathrm{Hg}\left(\mathrm{NO}_{3}\right)_{2}\right)\right.$ precipitando cloreto de mercúrio $\left(\mathrm{HgCl}_{2}\right)$. $\mathrm{O}$ teor de oxigênio foi calculado pela diferença entre o somatório do teor de $\mathrm{C}, \mathrm{H}, \mathrm{N}, \mathrm{S}$ e cinzas.

Por meio da análise imediata, foram determinados os teores de umidade, cinzas, matéria volátil e carbono fixo dos lodos. Esta análise foi realizada de acordo com a metodologia descrita pela norma ASTM 1131 (ASTM, 2003) com modificações (DOMENICO, 2013) no analisador termogravimétrico DTG-60 (Shimadzu, Japão). A análise consistiu na utilização de aproximadamente $40 \mathrm{mg}$ de amostra (com diâmetro de partícula $0,1 \mathrm{~mm}$ ), fluxo de gás de $100 \mathrm{~mL} \cdot \mathrm{min}^{-1}$ (gases $\mathrm{N}_{2}$ e ar sintético). A análise iniciou com gás $\mathrm{N}_{2}$ e a seguinte rampa: $50{ }^{\circ} \mathrm{C}$ durante $5 \mathrm{~min}$., posteriormente foi aplicada taxa de aquecimento de $50{ }^{\circ} \mathrm{C} \cdot \mathrm{min}^{-1}$ até $110{ }^{\circ} \mathrm{C}$ permanecendo durante $5 \mathrm{~min}$., depois foi aplicada taxa de aquecimento de $90{ }^{\circ} \mathrm{C} \cdot \mathrm{min}^{-1}$ até $950{ }^{\circ} \mathrm{C}$ permanecendo durante $15 \mathrm{~min}$. Na segunda etapa teve a entrada de ar sintético o sistema permaneceu na temperatura de $950{ }^{\circ} \mathrm{C}$ até que a perda de massa fosse constante. A determinação do poder calorífico foi realizada com base no modelo de Dulong (Buckley, T.J. 1991). Este modelo baseia-se na soma dos calores desprendidos pela combustão dos elementos que compõem o material (Equação 1), sendo este o poder calorífico superior da amostra (PCS). O poder calorífico inferior (PCI) é obtido pela diferença do PCS com a energia retida pela água na forma de vapor, formada pela combustão do hidrogênio e a umidade presente na amostra, conforme Equações 2 e 3.

$P C S=8100 . C+34400 .\left(H-\frac{o}{8}\right)+2500 . S$

$P C I=8100 . C+34400 .\left(H-\frac{o}{8}\right)+2500 . S-580 w$

$w=9 . H+u$

sendo:

PCS $=$ Poder Calorífico Superior $\left[\mathrm{kcal}_{\mathrm{kg}} \mathrm{kg}^{-1}\right]$

PCI = Poder Calorífico Inferior $\left[\mathrm{kcal}^{\mathrm{kg}} \mathrm{kg}^{-1}\right]$;

$\mathrm{C}=$ Fração mássica de carbono na amostra;

$\mathrm{H}$ = Fração mássica de hidrogênio na amostra;

$\mathrm{O}=$ Fração mássica de oxigênio na amostra;

$\mathrm{S}=$ Fração mássica de enxofre na amostra;

$\mathrm{u}=$ Fração de umidade; $\mathrm{w}=$ Água de combustão.

Termogravimetria: Para os ensaios termogravimétricos dos lodos LSC, LSAE, LSAN, LSANF e LSANS, aproximadamente $22,0 \mathrm{mg}$, previamente secas na balança de umidade, foram analisadas em uma balança termogravimétrica, sob atmosfera inerte (nitrogênio) e sob atmosfera oxidante (ar sintético), ambos a vazão de $0,5 \mathrm{~mL} \cdot \mathrm{min}^{-1}$. Onde foi aplicada uma taxa de aquecimento de $10{ }^{\circ} \mathrm{C} \cdot \mathrm{min}^{-1}$ 
até temperatura de $950{ }^{\circ} \mathrm{C}$. As amostras permaneceram na temperatura final da rampa até perda de massa constante. A temperatura de ignição foi determinada por comparação entre as curvas de perda de massa versus temperatura resultantes dos ensaios em atmosferas inerte e oxidante, correspondendo ao ponto de desvio entre as referidas curvas de perda de massa.

\section{RESULTADOS E DISCUSSÃO}

\subsection{Análise elementar e imediata}

Os resultados da análise elementar, imediata e poder calorífico dos lodos LSAE, LSAN, LSANS e LSANF está sendo apresentada na Tabela 2.

Tabela 2- Resultados das análises dos lodos sanitários e serragem de madeira.

\begin{tabular}{lcccc}
\hline Lodos & LSAE & LSAN & LSANS & LSANF \\
\hline Análise Elementar & & & & \\
\hline C [\%, b.s. ${ }^{1}$ ] & 33,87 & 23,66 & 20,15 & 25,51 \\
H [\%, b.s.] & 6,32 & 4,95 & 3,48 & 4,51 \\
N [\%, b.s.] & 5,88 & 3,15 & 2,74 & 3,74 \\
S [\%, b.s.] & 0,67 & 3,44 & 2,50 & 3,81 \\
O [\%, b.s.] & 24,64 & 18,85 & 19,79 & 19,08 \\
Cl [\%, b.s.] & 0,21 & 0,40 & 0,3 & 0,3 \\
\hline Análise Imediata & & & & \\
\hline Cinzas [\%, b.s.] & 28,40 & 45,55 & 52,04 & 43,05 \\
Matéria volátil [\%, b.s.] & 63,50 & 50,37 & 44,68 & 54,16 \\
Carbono fixo [\%, b.s.] & 8,08 & 4,08 & 3,27 & 2,78 \\
Umidade [\%, bruta] & 8,68 & 18,36 & 13,72 & 9,16 \\
\hline Poder Calorífico & \multicolumn{4}{c}{} \\
\hline PCS [b.s., MJ.kg ${ }^{-1}$ ] & 16,19 & 12,09 & 8,71 & 11,90 \\
PCI [b.s., MJ.kg ${ }^{-1}$ ] & 14,55 & 10,52 & 7,58 & 10,65 \\
\hline
\end{tabular}

${ }^{1}$ Base seca.

Entre as amostras de lodo avaliadas o LSAE apresentou maiores teores de carbono (elementar), hidrogênio e matéria volátil comparado às outras amostras, como foi apresentado na Tabela 2. De acordo com Virmond, et al. (2012), as concentrações de carbono e hidrogênio contribuem positivamente para o valor do PCS. O conteúdo de hidrogênio também influencia o poder calorífico inferior (PCI), devido à formação de água. $\mathrm{O}$ teor de oxigênio contribui negativamente em termos energéticos, porém, de forma positiva promovendo oxigenação homogênea do combustível, o que melhora a eficiência da conversão. Estes fatores justificam o maior valor de PCI obtido para essa amostra (14,55 MJ. $\left.\mathrm{Kg}^{-1}\right)$ quando comparada às demais.

A amostra LSAE apresentou a maior concentração de N (5,88 \%, b.s.). O nitrogênio em lodo de esgoto é proveniente da fração proteica deste material, que tem sua origem nos micro-organismos utilizados na purificação da água. Combustíveis com concentrações de $\mathrm{N}$ acima de $0,6 \%$, b.s. podem apresentar emissões de NOx durante a combustão que ultrapassem os limites da legislação 


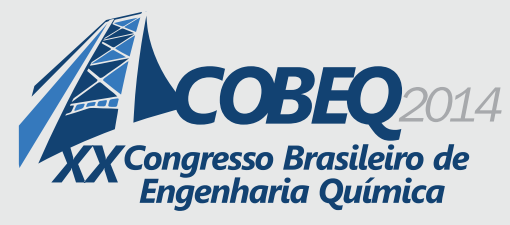

19 a 22 de outubro de 2014

Florianópolis/SC

(Obernberger, Brunner e Bärnthaler, 2006). Os lodos LSAN e LSANF apresentaram as maiores concentrações de enxofre (3,44 e 3,81\%, b.s.). Lodos de esgotos são característicos por apresentar concentrações altas de $\mathrm{S}$, e sua origem é atribuída aos compostos resultantes da decomposição das proteínas presentes nas fezes humanas, à presença de surfactantes e fertilizantes. E também o ambiente altamente redutor do tratamento anaeróbio na ETE, favorece a formação de enxofre elementar (Sígolo e Pinheiro, 2010). No caso do LSANF, foi encontrada maior concentração de enxofre devida a este lodo ser proveniente de dois processos: tratamento anaeróbio e flotação com de sulfato de alumínio. O enxofre presente no combustível, quando submetido à combustão, é oxidado a $\mathrm{SO}_{2}$, podendo resultar em problemas ambientais se sua emissão não for controlada. De acordo com Obernberger, Brunner e Bärnthaler, 2006), os problemas relacionados com as emissões de $S$ são esperados em concentrações acima de $0.2 \%$ (b.s.). Em relação ao teor de cloro, a amostra LSAN apresentou a maior concentração $(0,4 \%$ b.s.) em relação às demais. $O$ principal efeito da presença de $\mathrm{Cl}$ são o efeito corrosivo dos sais de cloro e $\mathrm{HCl}$ em peças de metal dos combustores, emissões de $\mathrm{HCl}$ que influenciam na formação de PCDD e PCDF. Estes problemas podem ser esperados para combustíveis com presença de cloro acima de 0.1\% (b.s.) (Obernberger, Brunner e Bärnthaler, 2006). Os lodos caracterizados apresentaram teores de matéria volátil, variando entre 39,73\% e 63,50\% (b.s.). O elevado teor de matéria volátil proporciona uma facilidade de ignição e um maior comprimento de chama. O carbono fixo é responsável pela manutenção e estabilidade da queima da amostra. O lodo LSAE apresentou a maior quantidade de carbono fixo, quando comparado aos demais lodos. Foi observada uma grande diferença no teor de cinzas (28,40 a 58,10\% b.s.) entre os lodos aeróbio e anaeróbios. O lodo LSAE apresentou o menor teor de cinzas, esta diferença ocorreu devido a influencia do sistema de tratamento dos lodos, sendo que amostras de lodo aeróbio possuem maior teor de matéria orgânica, e por consequência disto menor teor de cinzas que amostras provenientes de processos anaeróbios.

\subsection{Comportamento térmico}

As informações do comportamento térmico dos lodos, obtidas a partir das análises TG/DTG são importantes para a o ajuste dos parâmetros de operação dos processos de pirólise e combustão. Por meio dessa análise pode ser obtido o valor do combustível, as temperaturas iniciais e finais de degradação da biomassa, reatividade dos materiais carbonosos, a quantidade de cinzas e tempo total das reações. Os resultados da análise são dados como uma parcela de variação de perda de massa com a temperatura.

Em atmosfera inerte (pirólise), o material orgânico é submetido a uma série de reações complexas que geram produtos voláteis, matéria orgânica condensada e char. Em atmosfera oxidante (combustão) o processo é iniciado com a pirólise dos lodos, e produção de gás combustível e char. Os gases gerados queimam ao entrar em contato com $\mathrm{O}_{2}$. Finalmente, o char oxida com difusão de $\mathrm{O}_{2}$.

As curvas de perda de massa (TG) e taxas de perda de massa (DTG) obtidas durante a pirólise e combustão dos lodos LSAE (aeróbio) e LSAN (anaeróbio), estão sendo apresentadas nas Figuras 1 e 2, respectivamente. Os perfis dos demais lodos (LSANS e LSANF) estudados não foram apresentados, pois demostraram o mesmo comportamento que o lodo LSAN. 
Figura 1- Perfil termogravimétrico DTG (a) e TG (b) em atmosfera oxidante e atmosfera inerte do lodo LSAE.

(a)

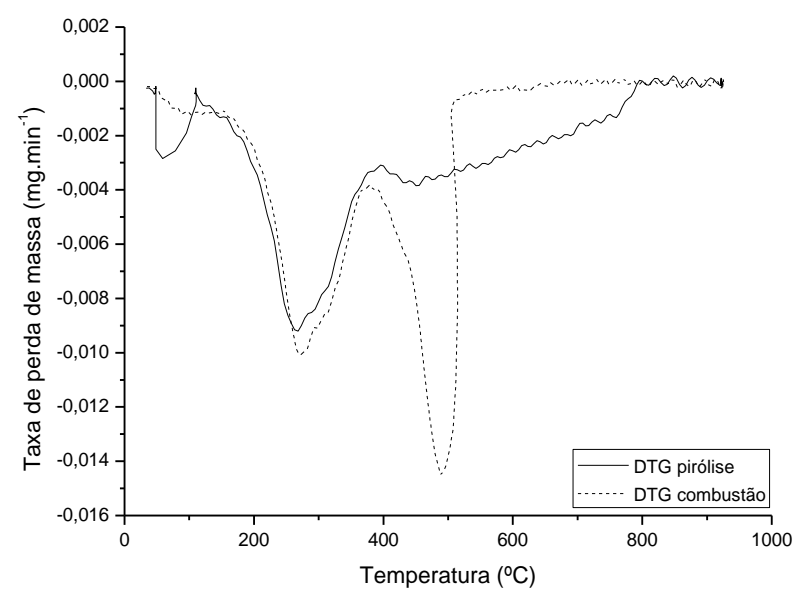

(b)

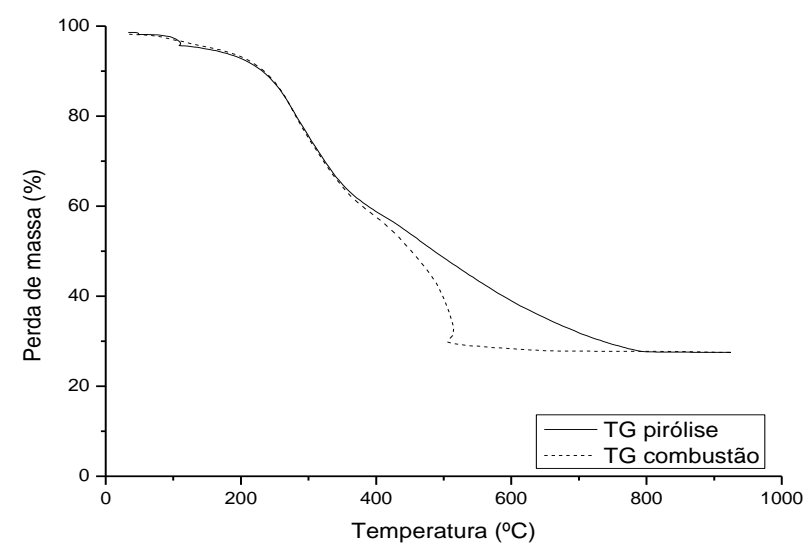

Figura 2 - Perfil termogravimétrico DTG (a) e TG (b) em atmosfera oxidante e atmosfera inerte do lodo LSAN.

(a)

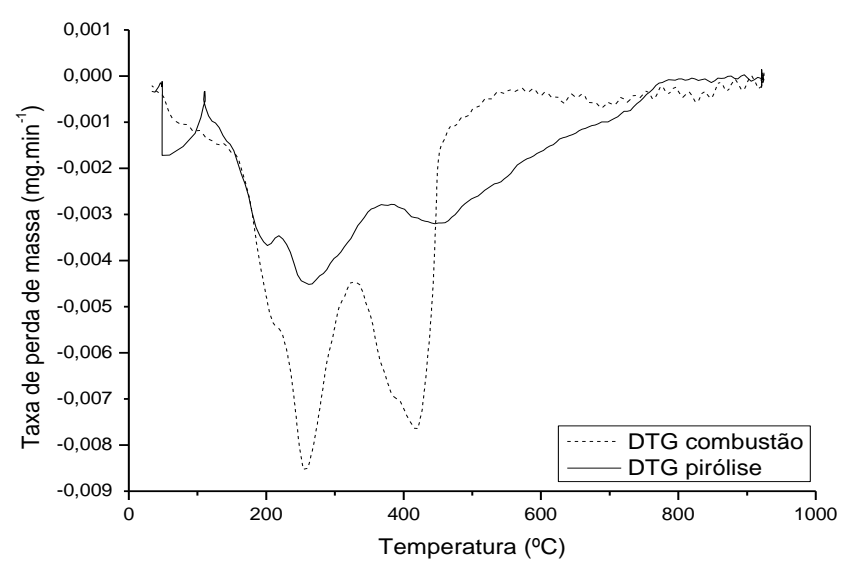

(b)

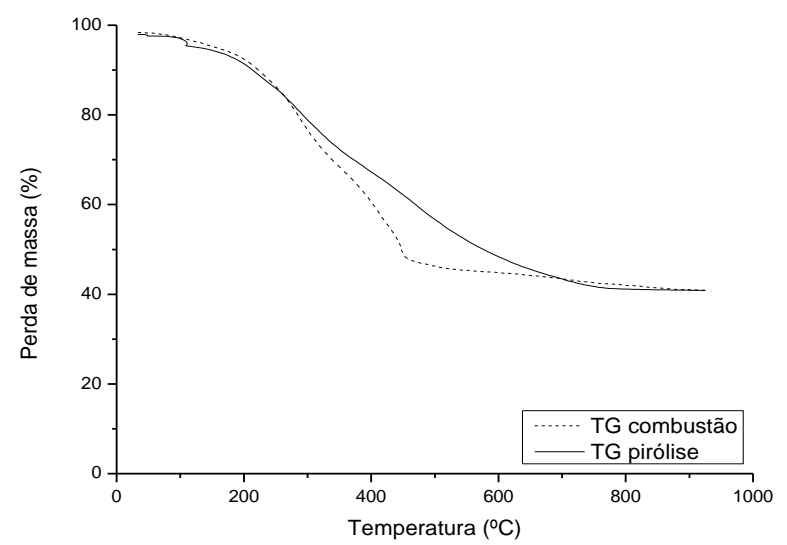

Nos perfis termogravimétricos (DTG) mostrados nas Figuras 1 e 2 (gráficos a), foram identificadas quatro etapas de perda de massa (BARNETO, et al., 2009). A primeira etapa (E1), esta associada com a perda de umidade e também a degradação dos compostos orgânicos de baixa estabilidade exemplos destes compostos são óleos, ceras e lipídios. Ocorre em baixas temperaturas, próximo a $100{ }^{\circ} \mathrm{C}$. A segunda etapa (E2) a fração degradada entre $115^{\circ} \mathrm{C}$ e $400{ }^{\circ} \mathrm{C}$ na pirólise e $150{ }^{\circ} \mathrm{C}$ e $375{ }^{\circ} \mathrm{C}$ na combustão. Esta etapa esta associada a volatilização de compostos relacionados a hemicelulose e celulose presentes no lodo. Na terceira etapa (E3) as frações degradam entre $400{ }^{\circ} \mathrm{C} \mathrm{e}$ $550{ }^{\circ} \mathrm{C}$ na pirólise e $375^{\circ} \mathrm{C}$ e $525^{\circ} \mathrm{C}$ na combustão. Esta fração está principalmente relacionada com a volatilização da lignina. O lodo por ser um resíduo urbano, pode conter outros compostos como os 


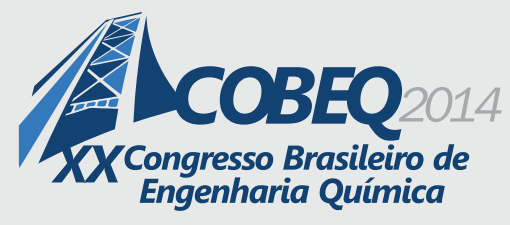

19 a 22 de outubro de 2014
Florianópolis/SC

plásticos, que degradam nessa faixa de temperatura. Plásticos são termicamente mais estáveis do que as amostras de papel (fração celulósica) e, como consequência, degradam a temperaturas mais elevadas, atingindo a taxa máxima de decomposição perto de $500{ }^{\circ} \mathrm{C}$. Sendo assim, em atmosfera inerte na etapa E2 ocorre a degradação da mistura de lignina-plástico (matéria orgânica não biodegradável). Em atmosfera oxidante, juntamente com a mistura é levado em conta à perda de massa da combustão do char. A degradação térmica da etapa E2 produz voláteis e char. Este char é acumulado no resíduo sólido e oxidado em uma faixa de temperatura que coincide com a etapa E3. Na quarta etapa (E4), uma fração foi degradada aproximadamente entre $625{ }^{\circ} \mathrm{C}$ e $725{ }^{\circ} \mathrm{C}$. Esta perda de massa está associada a compostos inorgânicos (carbonatos). A etapa E4 não foi observada no lodo LSAE.

Os lodos aeróbio e anaeróbio apresentaram curvas de TG distintas como foi mostrado nas Figuras 1 e 2 (gráficos b). O lodo aeróbio apresentou as curvas de TG da pirólise e combustão sobrepostas até a temperatura de aproximadamente $450{ }^{\circ} \mathrm{C}$, posteriormente, ocorreu um forte decaimento na curva de combustão. Os lodos anaeróbios apresentaram as curvas de TG da pirólise e combustão separadas, mas não muito longe. Estes dois comportamentos também foram observados por Font, et al. (2001) durante a decomposição térmica de vários tipos de lodos. Para os lodos aeróbios, ocorre a formação do char e depois a combustão do char na faixa de temperatura de $450{ }^{\circ} \mathrm{C}$ e $500{ }^{\circ} \mathrm{C}$. Neste caso, o oxigênio não acelera a decomposição térmica, fazendo com que as curvas de TG coincidam na primeira parte. Como consequência da formação do char, ocorre uma deterioração acentuada no final da curva devido à combustão do char. $\mathrm{O}$ fato do oxigênio não acelerar a decomposição térmica é devido ao tratamento aeróbio dos lodos. Neste tratamento a matéria orgânica torna-se estabilizada com a degradação de alguns dos compostos orgânicos, que reagiram facilmente com o oxigênio. Enquanto que a combustão dos lodos anaeróbios é considerada como pirólise oxidativa, ou pirólise acelerada, onde o oxigênio acelera a taxa de decomposição, e o char formado é queimado simultaneamente com a sua formação.

A perda de massa em atmosfera inerte do lodo aeróbio (52,95\% b.s.) foi menor do que em atmosfera oxidante $(72,34 \%$ b.s.). Os lodos anaeróbios (LSAN, LSANS e LSANF) também apresentaram menores perdas de massa em atmosfera inerte $(48,18 ; 42,35 ; 47,44 \%$ b.s.) quando comparada a atmosfera oxidante $(50,70 ; 44,87 ; 59,25 \%$ b.s.), respectivamente. As temperaturas de ignição dos lodos de esgoto sanitário variaram de 115 a $150{ }^{\circ} \mathrm{C}$. A temperatura e os mecanismos de ignição dependem da massa da amostra, tamanho da partícula, conteúdo de matéria volátil, concentração de oxigênio no combustível, bem como na composição da atmosfera (GROTKJAER, et al., 2003).

\section{CONCLUSÃO}

Os lodos de esgoto sanitário selecionados apresentaram bom potencial energético. O poder calorífico inferior dos lodos variou entre 7,58 MJ.kg-1 e 14,55 MJ.kg-1 (b.s.). O lodo aeróbio apresentou maior teor de matéria volátil e carbono fixo devido ao fato de ser mais rico em matéria orgânica, o que favorece sua conversão termoquímica. Por meio das análises termogravimétricas foi observado que, a decomposição térmica em atmosfera inerte e oxidante foi associada com a origem da amostra (anaeróbia ou aeróbia). Ambos os lodos apresentaram perda de massa em quatro picos. Para os 
lodos aeróbios, ocorreu a formação do char e posterior combustão do mesmo em temperaturas acima de $400{ }^{\circ} \mathrm{C}$, enquanto que para os lodos anaeróbios a combustão do char ocorreu de acordo com sua formação.

\section{AGRADECIMENTOS}

Os autores gostariam de agradecer ao CNPq, CAPES e a empresa Albrecht Indústria de Equipamentos Ltda pelo suporte financeiro e infraestrutura.

\section{REFERÊNCIAS}

BARNETO, A.G.; CARMONA, J.C.; ALFONSO, J.E.M.; BLANCO, J.D. Kinetic models based in biomass components for the combustion and pyrolysis of sewage sludge and its compost. Journal of Analytical and Applied Pyrolysis, v. 86, p. 108-114, 2009.

DOMENICO, MD. Gaseificação de Carvão Mineral Brasileiro na Presença de Ortossilicato de Lítio Visando a Produção Aumentada de Hidrogênio. Tese, UFSC, Florianópolis, 2013.

FONT, R.; FULLANA, A.; CONESA, J.A.; LLAVADOR, F. Analysis of the pyrolysis and combustion of different sewage sludges by TG. Journal of Analytical and Applied Pyrolysis, v. 58, p. 927 - 941, 2001.

GÓMEZ-RICO, M.F.; FONT, R.; FULLANA, A.; MARTÍN-GULLÓN, I. Thermogravimetric study of different sewage sludges and their relationship with the nitrogen content. J. Anal. Appl. Pyrolysis, v. 74, p. 421-42, 2005.

GROTKJÆR, T.; JOHANSEN-DAM, K.; JENSEN, A. D.; GLARBORG, P. An experimental study of biomass ignition.Fuel, v. 82, p. 825-833, 2003.

MAGDZIARZ, A.; WILK, M. Thermogravimetric study of biomass, sewage sludge and coal Combustion. Energy Convers. Manage. v. 75 p. 425-430, 2013.

OBERNBERGER, I.; BRUNNER, T.; BÄRNTHALER, G. Chemical properties of solid biofuels significance and impact. Biomass and Bioenergy, v. 30, p. 973-982, 2006.

RULKENS, W. Sewage sludge as a biomass resource for the production of energy: overview and assessment of the various options. Energy Fuels, v. 22, p. 9-15, 2008.

VIRMOND, E., DE SENA, R. F., ALBRECHT, W., AlThOFF, C. A., MOREIRA, R. F. P. M., JOSÉ, H. J., Characterisation of agroindustrial solid residues as biofuels and potential application in thermo- chemical processes. Waste Management, v. 32, n. 10, 1952-1961, 2012.

BUCKLEY, T.J. Calculation of higher heating values of biomass materials and waste components from elementals analyses and waste components from elementals analyses. Resources, Conservation and Recycling, v. 5, p. 329-341, 1991. 